\title{
LOCAL AND GLOBAL SUBORDINATION THEOREMS FOR VECTOR-VALUED ANALYTIC FUNCTIONS
}

\author{
BY \\ MARK FINKELSTEIN( ${ }^{1}$ ) AND ROBERT WHITLEY
}

Introduction. We extend the basic result on the subordination of analytic functions to analytic functions with values in a Banach space. The fundamental form of this theorem is as follows [2, p. 421]: Let $f$ and $g$ be two analytic (scalarvalued) functions defined on the unit disc $D$ with $g$ univalent and the range of $f$ contained in the range of $g$. Then $f$ and $g$ are analytically related, i.e. there is an analytic function $\omega$ mapping $D$ into itself with $f(z)=g(\omega(z))$ for all $z$ in $D$. This result extends to vector-valued analytic functions (for definitions see [1]) but there are subtleties: (1) the proof for scalar-valued functions consists of noting that $\omega(z)=g^{-1}(f(z))$ is analytic; for vector-valued functions not only is the proper notion of analyticity for $g^{-1}$ unavailable but, as we show below, $g^{-1}$ need not even be continuous, and (2) the result for scalar-valued functions is true for arbitrary domains (usually stated for $D$ only to avoid superfluous generality) while for vector-valued functions we cannot allow punctures in the domain of $g$.

The basic theorem on subordination has as a simple consequence the interesting result that if $f$ and $g$ are nonconstant analytic (scalar-valued) functions on $D$ with intersecting ranges then they are locally analytically related, i.e. there is a neighborhood $V$ in $D$ and an analytic function $\omega$ mapping $V$ into $D$ with $f(z)=g(\omega(z))$ for $z$ in $V$. This result also extends to vector-valued analytic functions but in this case the appropriate hypothesis is that $f$ and $g$ have ranges intersecting in an uncountable set. For $f$ and $g$ scalar-valued, if the ranges of $f$ and $g$ intersect at all, then they intersect in an uncountable set by the open mapping theorem; so, as in the basic theorem, it is the failure of the open mapping theorem for vector-valued analytic functions which lends interest to the generalization and makes the proof more delicate. We will show that for vector-valued $f$ and $g$ their ranges may intersect in a set containing an infinite number of accumulation points and yet not be locally analytically related.

\section{Global theorem.}

THEOREM. Let $X$ be a complex Banach space and $f$ and $g$ be two analytic functions mapping the unit disc $D$ into $X$. If $g$ is one-to-one with the range of $g$ containing the range of $f$, then there is an analytic function $\omega$ mapping the disc into itself with $f(z)=g(\omega(z))$ for all $z$ in $D$.

Received by the editors June 28, 1968 and, in revised form, January 27, 1969.

(1) Research supported in part by National Science Foundation Grant GP-7500. 
Proof. We may suppose that neither $f$ nor $g$ is constant, otherwise the result is immediate. The equation $g(\omega(z))=f(z)$ unambiguously defines $\omega$ as a map from $D$ into itself.

Let $W$ be the set of points in $D$ at which $\omega$ is analytic; $W$ is open by definition. We want to show that $W=D$. We will first show that $D-W$ is countable.

Assume that $D-W=W^{\prime}$ is uncountable. We can write $D=\bigcup K_{n}$, a countable union of compact sets. Then $W^{\prime} \cap K_{p}$ is uncountable for some index $p$ and is compact. Note that the function $\omega$ maps at most countably many points into a single point $a$, for if $\omega(K)=a$ with $K$ an uncountable set, then $g(a)=g(\omega(K))$ $=f(K)$ and $f$ is constant on an uncountable set. So, since such a set has an accumulation point, $f$ is constant throughout $D$ by the identity theorem. Hence $\omega\left(W^{\prime} \cap K_{p}\right)$ is uncountable and so has an uncountable number of accumulation points in $D$. Since $g^{\prime}$ can vanish at no more than a countable number of points in $D$, we can find $\omega_{0}$ an accumulation point of $\omega\left(W^{\prime} \cap K_{p}\right)$ with $g^{\prime}\left(\omega_{0}\right) \neq 0$. Then there are points $z_{n}$ in $W^{\prime} \cap K_{p}$ for which the sequence $\left\{\omega\left(z_{n}\right)\right\}$ converges to $\omega_{0}$. The sequence $\left\{z_{n}\right\}$ lies in a compact set and so by passing to a subsequence we may suppose that it converges to $z_{0}$. Since $g\left(\omega\left(z_{0}\right)\right)=f\left(z_{0}\right)=\lim f\left(z_{n}\right)=\lim g\left(\omega\left(z_{n}\right)\right)=g\left(\omega_{0}\right)$ and $g$ is one-to-one, we find that $\omega\left(z_{0}\right)=\omega_{0}$.

Let $x^{*}$ be a continuous linear functional on $X$ with $0 \neq x^{*} g^{\prime}\left(\omega\left(z_{0}\right)\right)=\left(x^{*} g\right)^{\prime}\left(\omega\left(z_{0}\right)\right)$. Since the derivative of $x^{*} g$ does not vanish at $\omega\left(z_{0}\right)$ there is a neighborhood $U$ of $\omega\left(z_{0}\right)$ with $x^{*} g$ one-to-one on $U$. Let $V$ be a neighborhood of $z_{0}$ such that $x^{*} f(V)$ $\subseteq x^{*} g(U)$. We define the analytic function

$$
h(z)=\left(x^{*} g\right)^{-1} x^{*} f(z) \text { for } z \text { in } V .
$$

For large enough $n, z_{n}$ is in $V$ and $\omega\left(z_{n}\right)$ is in $U$ and so $h\left(z_{n}\right)=\left(x^{*} g\right)^{-1} x^{*} f\left(z_{n}\right)$ $=\left(x^{*} g\right)^{-1} x^{*} g\left(\omega\left(z_{n}\right)\right)=\omega\left(z_{n}\right)$ and it follows that $g\left(h\left(z_{n}\right)\right)=f\left(z_{n}\right)$. By the identity theorem the analytic functions $g h$ and $f$ must agree on $V$. The function $g$ is oneto-one and $g(h(z))=f(z)=g(\omega(z))$ so $\omega(z)=h(z)$ is thus analytic on $V$. Hence $z_{0}$ is in $W$, a contradiction following from our assumption that $W^{\prime}=D-W$ was uncountable.

We now know that $W^{\prime}$ is countable and will use this fact to show that it is empty. Assume that $W^{\prime}$ is not empty. The set $W^{\prime}$ is relatively closed in $D$ and so is a locally compact Hausdorff space which is countable and then the Baire category theorem $\left[3\right.$, p. 85] tells us that $W^{\prime}$ must have an isolated point $y_{0}$. We now proceed to show that $\omega$ is analytic at $y_{0}$. Now $\omega$ is analytic in a deleted neighborhood $D_{0}$ of $y_{0}$ and is bounded on $D_{0}$, since the domain of $g$ is bounded, and thus $\omega$ has a removable singularity at $y_{0}$; let $\tilde{\omega}$ be $\omega$ redefined at $y_{0}$ so as to be analytic at $y_{0}$. Choosing points $\left\{y_{n}\right\}$ in the deleted neighborhood of $y_{0}$ which converge to $y_{0}$, we have $\left\{\tilde{\omega}\left(y_{n}\right)\right\}$ converging to $\tilde{\omega}\left(y_{0}\right)$. Noting that since $\tilde{\omega}$ is analytic and not constant, $\tilde{\omega}\left(D_{0}\right)$ is a (perhaps) deleted neighborhood of $\tilde{\omega}\left(y_{0}\right)$ and so $\tilde{\omega}\left(y_{0}\right)$ is in the domain of $g$ since the domain of $g$ has no punctures; hence $g\left(\tilde{\omega}\left(y_{0}\right)\right)=\lim g\left(\omega\left(y_{n}\right)\right)=$ $\lim f\left(y_{n}\right)=f\left(y_{0}\right)=g\left(\omega\left(y_{0}\right)\right)$. Because $g$ is one-to-one, $\omega\left(y_{0}\right)=\tilde{\omega}\left(y_{0}\right)$, i.e. $\omega$, alias $\tilde{\omega}$, 
is analytic at $y_{0}$. This is a contradiction since $y_{0}$ was not in $W$. Hence $D=W$. This completes the proof.

Notice that the only properties of the domain of $g$ which were used in the last paragraph of the above proof were that the domain be bounded and unpunctured. Clearly, the theorem holds when the domain of $g$ is conformally equivalent to such a domain, while the domain of $f$ is arbitrary.

To see that some restriction on the domain of $g$ is necessary, consider the following:

EXAMPLE 1. Let $X=C^{2}$, and

$$
\begin{array}{ll}
g(z)=\left(z(z-1), z(z-1)^{2}\right) & \text { on } 0<|z|<2 \\
f(z)=\left(z(z-1), z(z-1)^{2}\right) & \text { on }|z|<1
\end{array}
$$

Then $g$ is one-to-one, the range of $f$ is contained in the range of $g$, yet $f$ is not analytically related to $g$ (note that the domain of $g$ is punctured).

Note also that the function $f$ above provides us with an analytic, univalent function whose inverse is not continuous at $(0,0)$.

\section{Local theorem.}

THEOREM. Let $f$ and $g$ be analytic (vector-valued) on the unit disc D. Suppose the intersection of the ranges of $f$ and $g$ is uncountable. Then $f$ is locally analytically related to $g$. That is, there exists an analytic function $\omega(z)$ defined on a subdomain $D^{*}$ of $D$, mapping into $D$, such that $f(z)=g(\omega(z))$, all $z \in D^{*}$.

Proof. By excluding at most a countable discrete set from $D$ we may assume that $f^{\prime}$ and $g^{\prime}$ do not vanish on a domain $D_{1}$. The domain $D_{1}$ may be covered by a union of closed (compact) discs $K_{n}$ contained in $D_{1}$. Hence for some $K_{n}$ there are uncountably many $\zeta_{\alpha}$ in $K_{n}$, and for each such $\zeta_{\alpha}$ there is $z_{\alpha}$ in $D_{1}$ with $f\left(z_{\alpha}\right)=g\left(\zeta_{\alpha}\right)$. Hence the corresponding $z_{\alpha}$ form an uncountable set (since, arguing as in the proof of the global theorem, the set $\left\{g\left(\zeta_{\alpha}\right)\right\}$ is uncountable) from which can be extracted an uncountable subset $\left\{z_{\alpha^{\prime}}\right\}$ lying in some compact $K_{m}$. The corresponding $\left\{\zeta_{\alpha^{\prime}}\right\}$ in $K_{n}$, which is also an uncountable set, contain a convergent sequence $\left\{\zeta_{n}\right\}$ converging to $\zeta_{0}$ in $K_{n}$. From the corresponding $z_{n}$ in $K_{m}$ a convergent subsequence can be chosen. Now, after relabelings we have $z_{n} \rightarrow z_{0}, \zeta_{n} \rightarrow \zeta_{0}, f\left(z_{n}\right)=g\left(\zeta_{n}\right)$, and $f^{\prime}\left(z_{0}\right) \neq 0 \neq g^{\prime}\left(\zeta_{0}\right)$.

We now argue as in the global theorem. Let $x^{*}$ be any continuous functional with $x^{*} g^{\prime}\left(\zeta_{0}\right) \neq 0$. Then since $x^{*} f\left(z_{0}\right)=x^{*} g\left(\zeta_{0}\right)$, the function $\omega(z)=\left(x^{*} g\right)^{-1}\left(x^{*} f\right)(z)$ is analytic on a neighborhood $D^{*}$ of $z_{0}$ and for large $n$

$$
\omega\left(z_{n}\right)=\left(x^{*} g\right)^{-1} x^{*} f\left(z_{n}\right)=\left(x^{*} g\right)^{-1} x^{*} g\left(\zeta_{n}\right)=\zeta_{n} .
$$

Thus $g\left(\omega\left(z_{n}\right)\right)=f\left(z_{n}\right)$, for $n$ large enough. Hence $g \omega \equiv f$ on $D^{*}$ by the identity theorem.

We present an example to show that the hypothesis in the local theorem cannot be weakened. The basic idea is best seen in the following: 
ExAmple 2a. The range $X$ is $C^{2}$ and

$$
\begin{aligned}
& g(z)=\left(z(z-1), z^{2}(z-1)\right), \quad|z|<1, \\
& f(z)=\left(z(z-1), z^{2}(z-1) \cos \frac{2 \pi}{1-z}\right), \quad|z|<1 .
\end{aligned}
$$

Then

$$
f(1-1 / n)=g(1-1 / n) \rightarrow(0,0)=f(0)=g(0) .
$$

So the intersection of the ranges of $f$ and $g$ contain an accumulation point, yet $f$ is not locally analytically related to $g$. For if $f(z)=g(\omega(z))$ on some $D^{*}$ contained in $D$, then from the first component $\omega(z)=z$ or $\omega(z)=1-z$, contradicting $z^{2}(z-1) \cos (2 \pi /(1-z))=\omega(z)^{2}(\omega(z)-1)$ on $D^{*}$. Building on the above idea, we exhibit $f, g$ with ranges intersecting in a set with infinitely many accumulation points.

EXAMPLE 2b. Construct a sequence of points $\alpha_{n}$ on the unit circle with $0<\arg \alpha_{n}$ $<\pi / 3$, and with

(i) $\alpha_{n} \rightarrow 1 / 2+i(\sqrt{ } 3 / 2)$,

(ii) $\sum\left(1-\left|\alpha_{n}-1\right|\right)<\infty$.

Now for each $n$ choose a sequence $\left\{z_{k}^{(n)}\right\}$ with the properties

(i) $\left|z_{k}^{(n)}\right|<1, k=1,2,3, \ldots$,

(ii) $z_{k}^{(n)} \rightarrow \alpha_{n}$, as $k \rightarrow \infty$, and

(iii) $\sum_{k}\left(1-\left|z_{k}^{(n)}\right|\right)<2^{-n}, n=1,2,3, \ldots$

Then there exists [2, p. 240] a Blaschke product $B(z)$ with zeros at each $z_{k}^{(n)}$, and each $\alpha_{n}-1$.

We define the ( $C^{2}$-valued) functions $f, g$ as follows:

$$
g(z)=(\sin 2 \pi z, 0), \quad f(z)=(\sin 2 \pi z, B(z)) .
$$

Then $f\left(z_{k}^{(n)}\right)=g\left(z_{k}^{(n)}\right) \rightarrow\left(\sin 2 \pi \alpha_{n}, 0\right)=f\left(\alpha_{n}-1\right)=g\left(\alpha_{n}-1\right)$. And so the ranges intersect with infinitely many accumulation points.

If $f(z)=g(\omega(z))$, then comparing second coordinates, $B(z) \equiv 0$ on the domain of $\omega$, an obvious contradiction.

\section{REFERENCES}

1. N. Dunford and J. Schwartz, Linear operators, Part I, Interscience, New York, 1958.

2. E. Hille, Analytic function theory, Vol. II, Ginn, Boston, 1962.

3. J. Kelly and I. Namioka, Linear topological spaces, Van Nostrand, Princeton, N. J., 1963.

UNIVERSITY OF CALIFORNIA, IRVINE, CALIFORNIA 